\title{
Retrospective analysis of febrile patients reveals unnoticed epidemic of zika fever in Dielmo, Senegal, 2000
}

Fatim Ba ${ }^{1}$, Cheikh Loucoubar ${ }^{2}$, Ousmane Faye ${ }^{1}, G$ amou Fall ${ }^{1}$, Rose Nadege Penda Noelle Mbaye ${ }^{2}$, Mbacke Sembene $^{3}, M_{a w l o u t h}$ Diallo ${ }^{4}$, Aissatou Toure Balde ${ }^{5}$, Amadou Alpha Sall ${ }^{1}$ and Oumar Faye ${ }^{1 *}$

${ }^{1}$ Unite des Arbovirus et virus des fièvres hemorragiques, Institut Pasteur de Dakar, Senegal

${ }^{2}$ Groupe de Biostatistiques, Bioinformatique et Modelisation, Institut Pasteur de Dakar, Senegal

${ }^{3}$ Departement de Biologie Animale, Universite Cheikh Anta Diop de Dakar, Senegal

${ }^{4}$ Unite d'Entomologie, Institut Pasteur de Dakar, Senegal

${ }^{5}$ Unite d'Immunologie, Institut Pasteur de Dakar, Senegal

\begin{abstract}
Background: In Africa, recent malaria controls unveiled a large proportion of febrile illnesses of unknown origin. Few studies have been undertaken to identify these pathogens.

Research design and methods: We conducted a retrospective study to determine the seroprevalence of Zika virus (ZIKV) in a malaria endemic village in Senegal. We screened 487 samples collected from febrile individuals in 1999, 2000 and 2001 by using the enzyme-linked immunosorbent assay (ELISA) methods and the $90 \%$ plaque reduction neutralization tests (PRNT).

Results: Overall ZIKV IgG seroprevalence was 85.82\% (218/254) including 82.3\% (107/130) in 1999, 59.1\% (120/203) in 2000 and 92.2\% (142/154) in 2001. Patients aged 15-50 years old and over were the most affected ( $80 \%$ and $91 \%$ respectively) in contrast to patients under 15 (54\%) $(p<0.01)$.

None of the samples collected in 1999 and 2001 was found positive for ZIKV IgM. In 2000, 8.4\% (17/203) samples were positive for ZIKV IgM and 6.9\% (14/203) confirmed by PRNT. There was no ZIKV IgM positive in patients over 50 years old. Our analysis showed a strong association between Zika infections and nonmalaria febrile episodes $(p<0.01)$.
\end{abstract}

Conclusions: Our study revealed serological evidence of Zika epidemic fever in a malaria endemic area. These results highlight the importance of an effective ZIKV surveillance in Senegal.

\section{Introduction}

Zika fever is an acute viral disease transmitted by Aedes mosquitoes. Since its first isolation in Uganda in 1947, Zika virus (ZIKV) was reported in Africa, Southeast Asia and the Pacific causing sporadic cases and outbreaks [1,2]. Major outbreaks were described in Yap island, Micronesia in 2007 [3], French Polynesia in 2013-14 [4] and imported cases were reported in Europe and United States [5].

In 2015, a large outbreak occurred in Brazil where the virus was detected for the first time [6]. The infection was correlated with atypical symptoms like fetal microcephaly during pregnancy $[7,8]$. Severe neurological malformations and Guillain-Barre syndrome in adults have also been reported. From Brazil, the virus spread to other countries in South America and Central America. In addition, new modes of transmission including maternal-fetal transmission and sexual transmission have been described [9-11]. This, correlated with the geographic expansion of the virus and its endemicity in many countries, drew attention to ZIKV as being a public health emergency of international concern.

ZIKV belongs to the Flaviviridae family, genus Flavivirus, and isolates analyzed so far could be classified as having three lineages: Asian, East and West African [12].

ZIKV infection is generally a mild and short-lasting illness with fever, maculopapular rash, arthralgia, headache, myalgia and joint pains. In addition to the common clinical presentation, severe ZIKV infections associated to Guillain-Barre syndrome and neurological malformations such as microcephaly specifically on newborns were described $[4,13]$.

The virus is transmitted to humans and non-humans primates by Aedes spp. mosquitoes. Wild mosquito species such as Aedes africanus, Ae. luteocephalus, Ae. taylori, Ae. vittatus and Ae. furcifer were frequently involved in the sylvatic cycle in Africa [14-16]. Occasionally, ZIKV transmission occurs in a peri-domestic or urban environment between humans and domestic Aedes aegypti. Aedes albopictus was recently found to be susceptible to ZIKV [17] and could be a probable outbreak vector [18]

In Africa, ZIKV was first isolated from monkeys in Uganda in 1947. In 1948 the virus was reported in mosquitoes and humans in Nigeria [19]. Thereafter, serological and entomological surveys showed its larger

Correspondence to: Dr. Oumar Faye, Unite des Arbovirus et virus des fièvres hemorragiques, Institut Pasteur de Dakar, Dakar, Senegal, Tel: 002217797871 01, Fax: 002213383992 10, E-mail: oumarfaye@pasteur.sn

Key words: flavivirus, malaria, senegal, seroprevalence, west africa, zika

Received: January 22, 2018; Accepted: February 01, 2018; Published: February 19,2018 
distribution in the continent, in the western areas (Gabon, Cameroon, Nigeria Ivory coast, Senegal) and eastern areas (Somalia, Tanzania, Ethiopia) [14-16] where its potential epidemic vectors, Aedes aegypti and Aedes albopictus were present. In 2007, Zika epidemic occurred in Gabon, the virus was circulating in humans and Aedes albopictus mosquitoes in urban settings. This was the first isolation of ZIKV in the Asian tiger mosquitoes [18]. In 2016, Zika outbreak spread from South America to Cabo Verde islands leading to first microcephaly cases linked to ZIKV in Africa [12]. This also corresponds to the first Zika outbreak linked to the Asian lineage in the continent. In July 2016, Guinea-Bissau, West Africa, recorded Zika infection cases linked to the African lineage [12].

In Senegal, entomological and virological surveillance in the Southeastern country showed a sylvatic circulation of ZIKV since 1968 between Aedes mosquitoes (Ae. furcifer, Ae. Luteocephalus and Ae. vitattus) and Cercopithecus aethiops, Erythrocebus patas monkeys [20]. Sylvatic mosquitoes including Culex perfuscus, Mansonia uniformis and Anopheles coustani were also found infected by the virus [16]. The competence of these local species to transmit the virus is though not well known. In humans, yearly sporadic cases have been described. Seroprevalence and direct detection of ZIKV in humans have been reported since 1969 during surveillance programs in the West (Dakar, Bandia, Mekhé) and in the Center (Diourbel) of Senegal [20].

ZIKV surveillance in Africa is poorly reported. Despite its large distribution in Africa, few laboratories have the capacity of identifying the virus, which leads to unnoticed circulation of Zika. Malaria and flaviviruses such as Dengue or Chikungunya induced similar clinical symptoms with Zika virus infection. They are widely detected in Africa where ZIKV remained unnoticed.

Since 1990, a cohort study on malaria was conducted in Senegal, in Dielmo village to understand the transmission dynamics and the determinants of the disease. Therefore, the population of the village is being followed in the biological, entomological and immunological parameters. A cross-sectional sampling of the whole village was done annually and through that process a large amount of samples and data were available and helped establish a large database and sera bank [21].

Malaria control programs in Dielmo have not only led to a drastic reduction of disease prevalence [22] but also to the uncovering of other causes of fever presumably believed as malaria. These febrile episodes may be caused by other infectious pathogens including bacteria, parasites or viruses $[23,24]$.

To date, few studies focus on a probable role of arboviruses infections in these febrile diseases in Senegal.

A retrospective pilot study on the human samples of the Dielmo project (a study on the natural history and determinants of malaria infection) showed interestingly a circulation of flaviviruses in the population of Dielmo in 2000 (Data not published); ZIKV serosurveys were not included in this prior study.

The purpose of this paper is to report a retrospective study using samples collected between 1999 and 2001 to appreciate the circulation of ZIKV and to evaluate its implication in non-malaria fever cases.

\section{Patients and methods}

\section{Ethics statement}

This study is a clinical monitoring of pathologies in the villages of Dielmo Ndiop in order to identify the origin of non-malaria fever for better understanding of fevers. In this context, an authorization under the number $00112 \mathrm{MSP} / \mathrm{DS} / \mathrm{CNERS}$ was obtained from the Senegalese National Ethical Committees for Health Research for a preliminary screening of the serum bank for which the results are presented in this present study. Written informed consent was obtained from all adult participants and from parents or legal guardians of minors under the age of 18 years. The data were recorded in a confidential database, all the samples were anonymous and only reference numbers were used during the analysis.

\section{Study area}

Dielmo (13" $\left.45^{\prime} \mathrm{N}, 16^{\prime \prime} 25^{\prime} \mathrm{W}\right), 270 \mathrm{~km}$ southeast from Dakar is a rural area located in the Central West of Senegal (Figure 1). The main activity is agriculture and the rainy season lasts from the end of June to mid-October. A permanent river, the Nema, which promotes a perennial and high rate malaria transmission, borders the village. The population is distributed over two residential groupings with a distance of $300 \mathrm{~m}$ between the groupings, the original residential grouping is Dielmo itself, where most of the population is located, and the more recent residential grouping called Santhiou Mouride. Each settlment (huts belonging to same family) was identified by the letter D followed by a number (for example D10). Dielmo and Santhiou Mouride included 35 settlements at the time of the study.

\section{Study participants}

This retrospective study was conducted on human sera from the Dielmo project. This project consists on a longitudinal malaria survey in Dielmo and Ndiop since 1990 in which cross-sectionals sampling of the whole village was done annually. Each participant was sampled once yearly, corresponding to one sample per participant per year over three years. However, some participants were not present for one or two yearly cross sectionals.

We selected samples from February 1999 to November 2001 cross sectional surveys in Dielmo based on the following criteria: all febrile cases, malaria positive and malaria negative cases. Once collected, samples were conserved at $-20^{\circ} \mathrm{C}$. Data include individual's information such as age, sex, family status, some clinical symptoms and malaria diagnosis.

\section{Laboratory procedures}

All the experiments were performed at the Institute Pasteur de Dakar. All the samples were systematically screened for enzyme-linked immunosorbent assay (ELISA) IgG and IgM against Zika viruses and a $90 \%$ plaque-reduction neutralization test (PRNT) was done only on the positive. Moreover, the ZIKV positive samples were also tested against other flaviviruses such as yellow fever virus (YFV), dengue virus (DENV) and West Nile virus (WNV) by the ELISA and the PRNT method.

\section{ELISA}

IgG and IgM antibodies against ZIKV and other flaviviruses were detected in sera by specific in-house ELISA method adapted from previous protocols [25-27]. Positive and negative controls sera for ELISA are prepared and provided by the WHO collaborating Center at the Institute Pasteur of Dakar in Senegal. As positive controls, human sera tested positive to ELISA and PRNT with no cross-reactions to other flaviviruses was used. For negative control, antibody-negative serum was used.

\section{ELISA IgM}

We determined the presence of DENV, YFV, WNV and ZIKV IgM in our samples by the capture enzyme-linked immunosorbent assay 


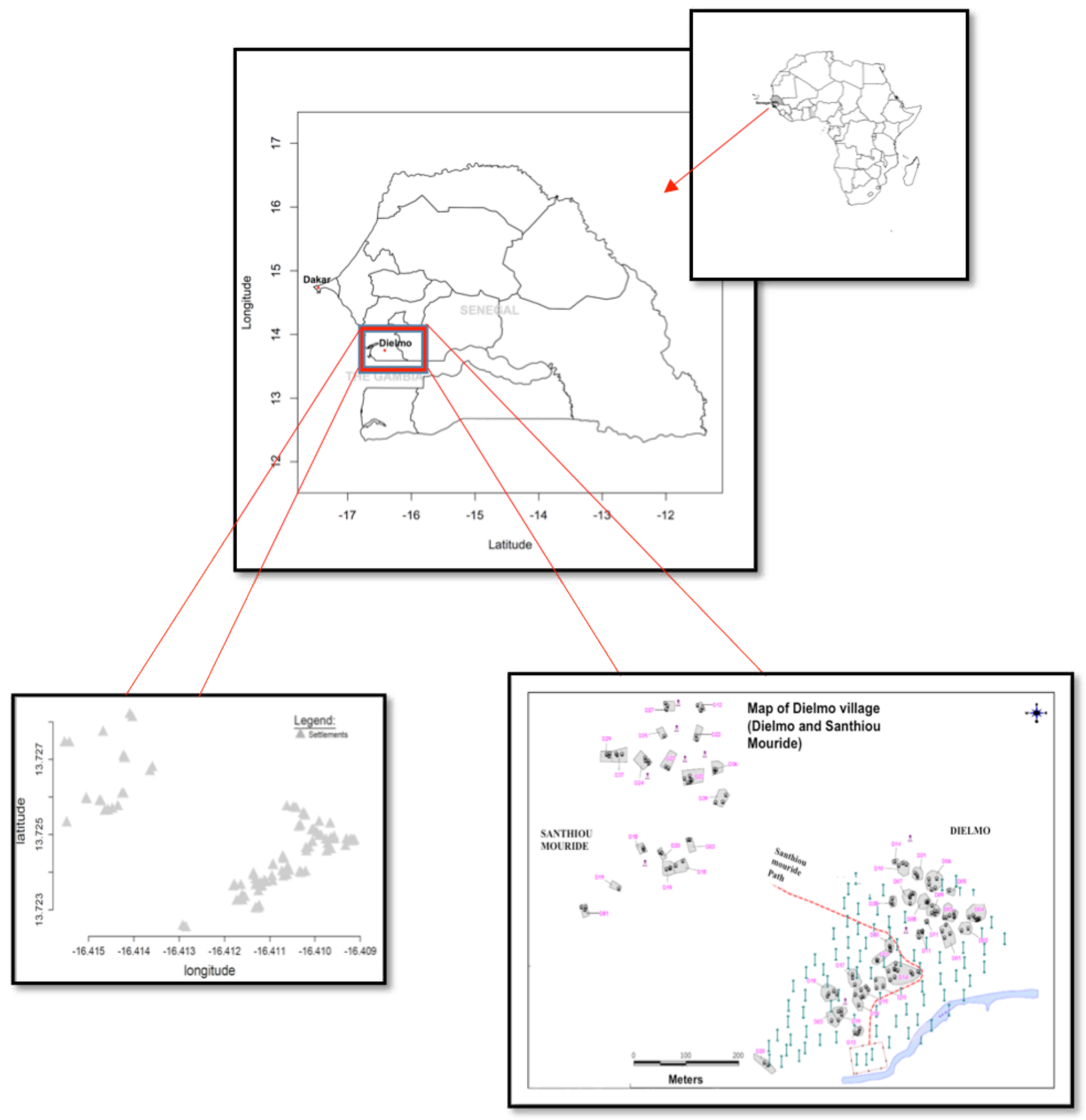

Figure 1. Location of the study site (Dielmo).

(MAC ELISA) routine. A 96 well microtiter plate was coated with a monoclonal capture antibody (anti human IgM) and incubated at $4^{\circ} \mathrm{C}$ overnight. Human sera were heat-inactivated $\left(56^{\circ} \mathrm{C}, 30 \mathrm{mn}\right)$ and screened at a dilution of 1:100 in phosphate-buffered saline (PBS) plus $0.05 \%$ Tween plus $1 \%$ milk powder. After washing the plate three times with PBS plus $0.05 \%$ Tween, serum samples and controls were added on duplicate to the plate and incubated at $37^{\circ} \mathrm{C}$ for $1 \mathrm{hr}$. Wells were washed three times, specific and non-specific antigens were deposited, and the plate was incubated $1 \mathrm{hr}, 37^{\circ} \mathrm{C}$. After washing as before, specific immune ascite was added to each well. After incubation and washing, a conjuguate (peroxidase labeled antibody specific to mouse IgG) was added and allowed to react for $1 \mathrm{hr}, 37^{\circ} \mathrm{C}$. A tetra-methylbenzidine (TMB) substrate was added to the IgM conjugate complex, and the coloration reaction was stopped with sulphuric acid.

The intensity of the coloration is proportional to the level of virus specific antibodies present in the serum. An ELISA microplate reader 
shows the Optical Density (OD) of the absorbance, an OD unit $\geq 0.2$ was defined as a positive IgM.

\section{ELISA IgG}

For ELISA IgG antibodies detection, a 96 well plate was coated with a mouse hyper immune ascitic fluids and incubated at $4^{\circ} \mathrm{C}$ overnight. After washing 3 times with PBS plus $0.05 \%$ Tween, specific antigen was added, and the plate was incubated $1 \mathrm{hr} 37^{\circ} \mathrm{C}$. Serum samples were added, and specific binding was revealed by an anti-Human IgG horseradish peroxidase conjugated. Optical density values were determined as above.

\section{PRNT}

Positive IgG or IgM ELISA sera were confirmed by an in-house PRNT test adapted from the published standard procedures to rule out cross reaction between ZIKV and other related flaviviruses [28,29].

The PRNT90 assay was performed using PS (Porcine Kidney Cells) cell lines and a semi solid carboxymethylcellulose (CMC) overlay. All sera were inactivated at $56^{\circ} \mathrm{C}$ for $30 \mathrm{mn}$ and then screened for specific neutralizing antibodies.

We used the ZiKV ref. MR766, YFV ref. 17D, WNV ref. EG101 and DENV New Guinea C (NGC) reference strains. For each virus, we prepared virus stock at 1000 Plaque Forming Units (PFU)/ml. We determined from the viral stock serial virus dilutions (viral range) capable of neutralizing 50 to $99 \%$ of the virus.

Sera were heat-inactivated and diluted at 1:10 for a qualitative PRNT or at serial twofold dilutions (1:10 to 1:640) for a quantitative PRNT. Sera were then mixed to an equal volume of the viral stock and distributed in the plates wells. A suspension of confluent PS cells was then added to the wells containing the mixture and those containing the viral range. After a $4 \mathrm{~h}$ incubation at $37^{\circ} \mathrm{C}$, plates were overlaid with CMC solution and placed back in the $37^{\circ} \mathrm{C}$ incubator for four to five days. After washing and coloration with amido black, plaques were counted and PRNT90 titers were calculated as the inverse of the maximum serum dilution capable of neutralizing $90 \%$ or more of the tested virus.

\section{Statistical analyses}

Statistical analyses were performed using STATA (version SE/11.0) and R (version 3.1.1 (2014-07-10)) statistical software. Fisher exact test was performed to compare seroprevalence between years and to test association between Zika infections and occurrence of non-malaria febrile episodes.

\section{Results}

In Dielmo, we selected 487 sera samples from 254 febrile patients, during the 1999, 2000 and 2001 cross sectional surveys and mainly the ones that were sampled from October to December. This time frame corresponded to an increased level of fever not related to malaria in Dielmo. Moreover, at the same time period it was observed a high circulation of flaviviruses in Senegal.

Sera based on the previous criteria were screened for Zika IgM and IgG antibodies. Among the 487 sera, 130 (26.7\%) were sampled in 1999, $203(41.7 \%)$ in 2000 and $154(31.6 \%)$ in 2001 (Table 1). Demographical data were not available for 8 individuals. There were 132 males and 114 females (M/F sex ratio: 1.16). The median age was 28 years (range $4-89$ ).

\section{ZIKV IgM results}

None of the 130 and 154 samples collected respectively in 1999 and 2001 was found positive for ZIKV IgM. Among patients sampled in 2000, 8.4\% (17/203) were tested positive for ZiKV IgM by the ELISA method (Table 1).

Seroneutralisation test (PRNT) confirmed 14 of the 17 ZIKV positive samples, showing a prevalence of $6.9 \%$ (14/203) for ZIKV IgM. Analysis by age and sex of confirmed IgM positive cases (Table 2) showed higher rates in children under 15 years old (11.8\%) compared to young adults aged $15-50$ years $(5.1 \%)$, however those variations were not significant $(p=0.2014)$. No Individual was tested positive to ZIKV IgM in patients over 50 years old. Seropositivity was similar between female $(8.7 \%)$ and male $(4.8 \%), p=0.274$.

\section{ZIKV IgG results}

The overall ZIKV IgG seroprevalence was $85.82 \%(218 / 254)$ including $82.3 \%(107 / 130)$ in $1999,59.1 \%(120 / 203)$ in 2000 and $92.2 \%$ $(142 / 154)$ in 2001 . Patients aged $15-50$ years and more than 50 years old were the most affected $(80.4 \%$ and $91.5 \%$ respectively) in contrast to patients under 15 years old (54\%), $p<0.01$ (Table 1).

There was a significant IgG positive samples increase between 2000 and $2001(p<0.0001)$ (Table 1, Figure 2). This IgG increase was stronger among the patients under 15 years old than in the other age groups: $53 \%$ (23/43) of positive cases in 2000 and $100 \%$ (45/45) in 2001, $p<0.01$ (Figure 3).

Beside these previous analysis, we looked at the follow up of confirmed IgG antibodies within the 14 confirmed ZIKV IgM individuals (Table 3). In 2000, 9 out of the 14 individuals did not develop any ZIKV IgG antibodies, one (1) individual was tested negative by the PRNT and only 4 were confirmed ZIKV IgG positive. Among the 4 positive, neutralizing antibody titers against other tested flaviviruses (YFV, DENV and WNV) showed that one indivual had confirmed WNV IgG antibodies and another individual had both YFV and WNV IgG antibodies. ZIKV neutralizing antibody titers was higher in this last individual ( $>640)$ than the YFV (1:20) and the WNV (1:320). In 2001, 10 out of the 14 confirmed ZIKV IgM individuals were screened for the presence of confirmed ZIKV IgG; the 4 remaining individuals were not present during the cross sectionals of 2001. Among the 10 individuals, 8 developed confirmed ZIKV IgG antibodies and only 3 of them had neutralizing IgG antibodies against another flavivirus (YFV, DENV and WNV). Except for YFV, the neutralizing antibody titers against ZIKV were at least two-fold higher.

\section{Temporal analysis and spatial distribution pattern of the patients}

We performed a temporal and spatial distribution pattern of the 14 confirmed ZIKV IgM cases. Patients tested positive to ZIKV IgM were sampled in October and November 2000, with 1 and 13 positive cases respectively.

The spatial distribution of these positive cases showed that most of them were located in settlements next to the Nema river. Analysis by settlement showed that 10/35 settlements were affected with 5/14 cases of Zika virus infection found in D11 and D16 settlements located next to the main village path. Some cases were found in Santhiou Mouride, a more recent extent of the village of Dielmo (Figure 4). 


\section{Clinical associations of Zika virus infection}

Clinical manifestations by the time of sample collection were not available. This was due to the fact that sampling was done during crosssectional and febrile episodes could occur at any time during the year. Analyses were performed based on the symptoms of episodes appearing up to three months before the sampling time. The symptoms that were present during febrile episodes were fever, headache, abdominal pain,

Table 1. Demographic and serological characteristics of the study participants*

\begin{tabular}{|c|c|c|c|c|}
\hline & & \multicolumn{3}{|c|}{ Serological results } \\
\hline YEAR & & $\begin{array}{c}\text { \# Tested } \\
\text { samples }\end{array}$ & \# ZIKV IgM+ & $\begin{array}{c}\text { \# ZIKV } \\
\text { IgG+ }\end{array}$ \\
\hline 1999 & 130 & 130 & 0 & 107 \\
\hline 2000 & 203 & 203 & 17 & 120 \\
\hline 2001 & 154 & 154 & 0 & 142 \\
\hline SEX & & & & 183 \\
\hline Male & 132 & 245 & 6 & 179 \\
\hline female & 114 & 234 & 10 & 7 \\
\hline unknown & 8 & 8 & 1 & 66 \\
\hline AGE GROUP & & & & 221 \\
\hline$\leq 15$ & 76 & 122 & 10 & 75 \\
\hline $15-50$ & 136 & 275 & 6 & 7 \\
\hline$>50$ & 34 & 82 & 0 & 1 \\
\hline unknown & 8 & 8 & & \\
\hline
\end{tabular}

Table 2. Distribution of ZIKV IgM confirmed patients (PRNT) by age and sex, in 2000

\begin{tabular}{|c|c|c|}
\hline Characteristic & Number tested & Percent \\
\hline Year & & \\
\hline 2000 & 203 & $6.9 \%$ \\
\hline Sex & & \\
\hline Male & $5 / 103$ & $4.8 \%$ \\
\hline Female & $8 / 92$ & $8.7 \%$ \\
\hline Unknown & $1 / 8$ & - \\
\hline Age group & & \\
\hline$\leq 15$ & $8 / 68$ & $11.8 \%$ \\
\hline $15-50$ & $5 / 98$ & $0.1 \%$ \\
\hline$>50$ & $0 / 29$ & - \\
\hline Unknown & $1 / 8$ & \\
\hline Total & $\mathbf{1 4}$ & $0 \%$ \\
\hline
\end{tabular}

Table 3. Neutralizing IgG antibody profile of the 14 confirmed Zika IgM individuals in 2000 and 2001.

\begin{tabular}{|c|c|c|c|c|c|c|c|c|}
\hline \multicolumn{7}{|c|}{ 2000 } & \multicolumn{5}{c|}{ 2001 } \\
\hline & \multicolumn{9}{|c|}{ InNividuals } & ZIKV & YFV & DENV & WNV & ZIKV & YFV & DENV & WNV \\
\hline $03 / 05$ & $1: 640$ & - & - & $>640$ & 0 & - & - & - \\
\hline $08 / 15$ & 0 & - & - & - & $1: 160$ & - & - & - \\
\hline $09 / 12$ & 0 & - & - & - & $1: 80$ & - & - & - \\
\hline $10 / 07$ & 0 & - & - & - & $1: 80$ & $1: 320$ & - & - \\
\hline $11 / 03$ & 0 & - & - & - & $>640$ & - & $1: 80$ & - \\
\hline $11 / 10$ & 0 & - & - & - & $1: 80$ & - & - & $1: 10$ \\
\hline $16 / 07$ & $>640$ & $1: 20$ & - & $1: 320$ & $1: 320$ & - & - & - \\
\hline $16 / 22$ & 0 & - & - & - & $1: 40$ & - & - & - \\
\hline $16 / 23$ & NC & - & - & - & $1: 40$ & - & - & - \\
\hline $17 / 17$ & 0 & - & - & - & Nc & - & - & - \\
\hline $18 / 16$ & $1: 80$ & - & - & - & 0 & - & - & - \\
\hline $20 / 11$ & 0 & - & - & - & Nc & - & - & - \\
\hline $22 / 05$ & 0 & - & - & - & Nc & - & - & - \\
\hline Unknown1 & $1: 20$ & - & - & - & Nc & - & - & - \\
\hline
\end{tabular}

Note: (0) Tested negative by ZIKV IgG Elisa, (-) tested negative by YFV, WN and DENV $\mathrm{IgG},(\mathrm{NC})$ not confirmed by PRNT method, $(\mathrm{Nc})$ not collected during cross sectional surveys.

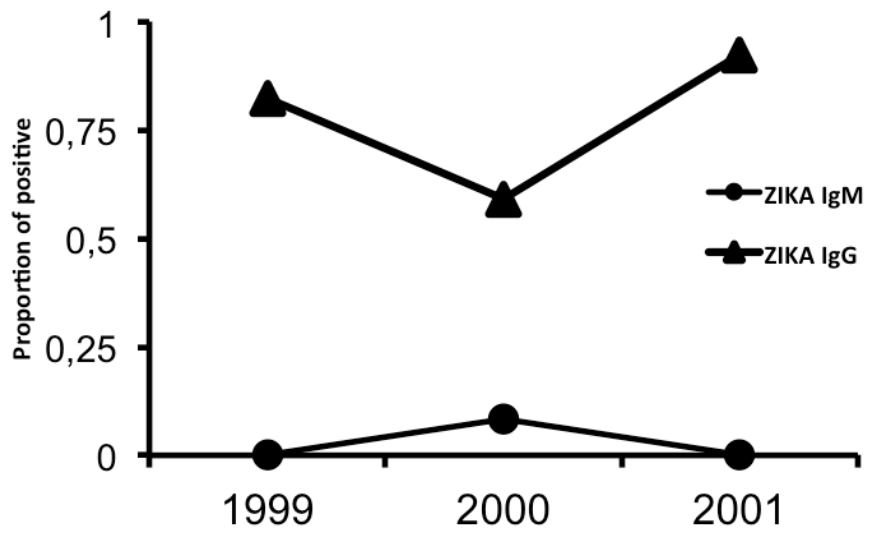

Figure 2. Evolution of Zika positive Elisa IgG and IgM from 1999 to 2001.

cough, asthenia, vomiting and diarrhea. All these different symptoms were found in both individuals tested Zika positive and Zika negative IgM during all febrile episodes occuring up to 3 months before the sampling time (Figure 5).

Association of Zika infection and fever was significant in the period 15 days to 3 months before the date of sample collection $(p=0.013$ at $<$ 15 days, and $p<0.001$ at $<45$ days to $<90$ days). In contrast, diarrhea was significantly not associated to the infection: it was present in noninfected persons as early as 45 days before the date of collection and up to 90 days ( $p=0.031$ at $<45$ days, $p=0.014$ at $<60$ days and $<75$ days, $p=0.015$ at $<90$ days $)$.

\section{Proportion of non-malaria febrile episode among recent Zika virus infected and non-infected patients}

We were interested in this part of the study to see whether the nonmalaria febrile episodes observed during our period study could be related to the infection with ZIKV. Thus, we analyzed the proportion of non-malaria febrile episodes in two groups: confirmed positive Zika IgM positive and non-positive Zika IgM in the study population in 2000 (Table 4).

Association between recent ZIKV infections and the rate of nonmalaria febrile episodes was more significant when only episodes occurring within the 15 days before the positive Zika diagnosis were considered. The association decreased with inclusion of further episodes and remained significant until two months but ceased with inclusion of episodes beyond this delay $(p=0.03)$.

\section{Discussion}

Few arboviral epidemiological studies in Dielmo have been reported in the literature. Surveillance of arboviruses in Senegal is mainly conducted in the southeastern of Senegal where a regular investigation of yellow fever and related flaviviruses in mosquitoes and humans is carried out since the 1970s. Since then, ZIKV were reported mainly during epizootic amplification from wild Aedes mosquitoes and sporadic human cases have been described.

To our knowledge, no study has been reported in Dielmo to analyze the seroprevalence of anti-Zika virus antibodies in the human population. Our retrospective study has revealed serological evidence of a ZIKV outbreak in humans in Senegal in 2000 and implication of ZIKV infections in non-malaria fever episodes. Indeed, the presence of ZIKV specifics IgM antibodies were found in human sera indicating recent infection; specific IgM can be detected in the earlier days 


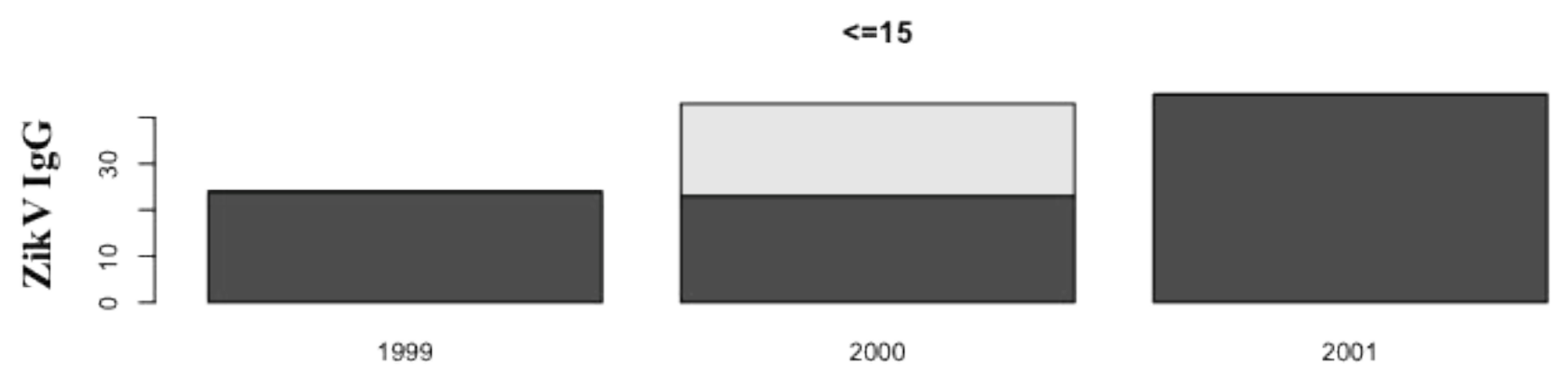

$15-50$
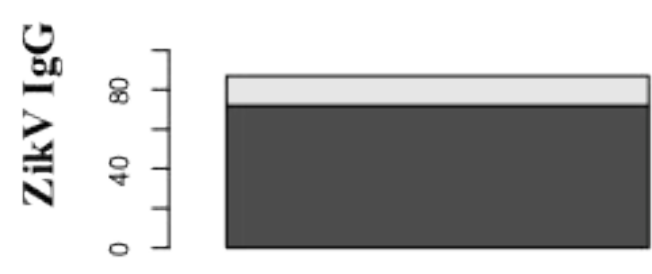

1999

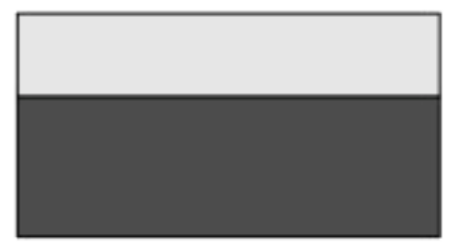

2000

$>50$
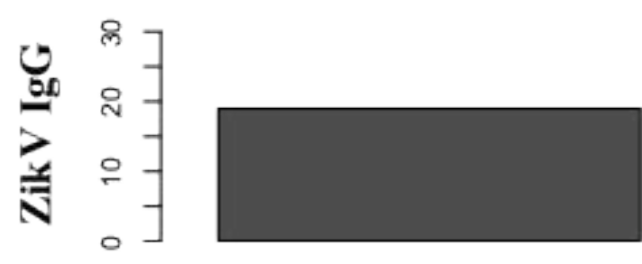

1999

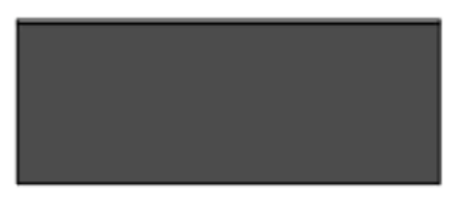

2001

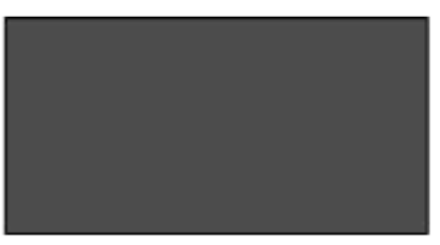

2001

Figure 3. Evolution of positive Elisa IgG by age and year.

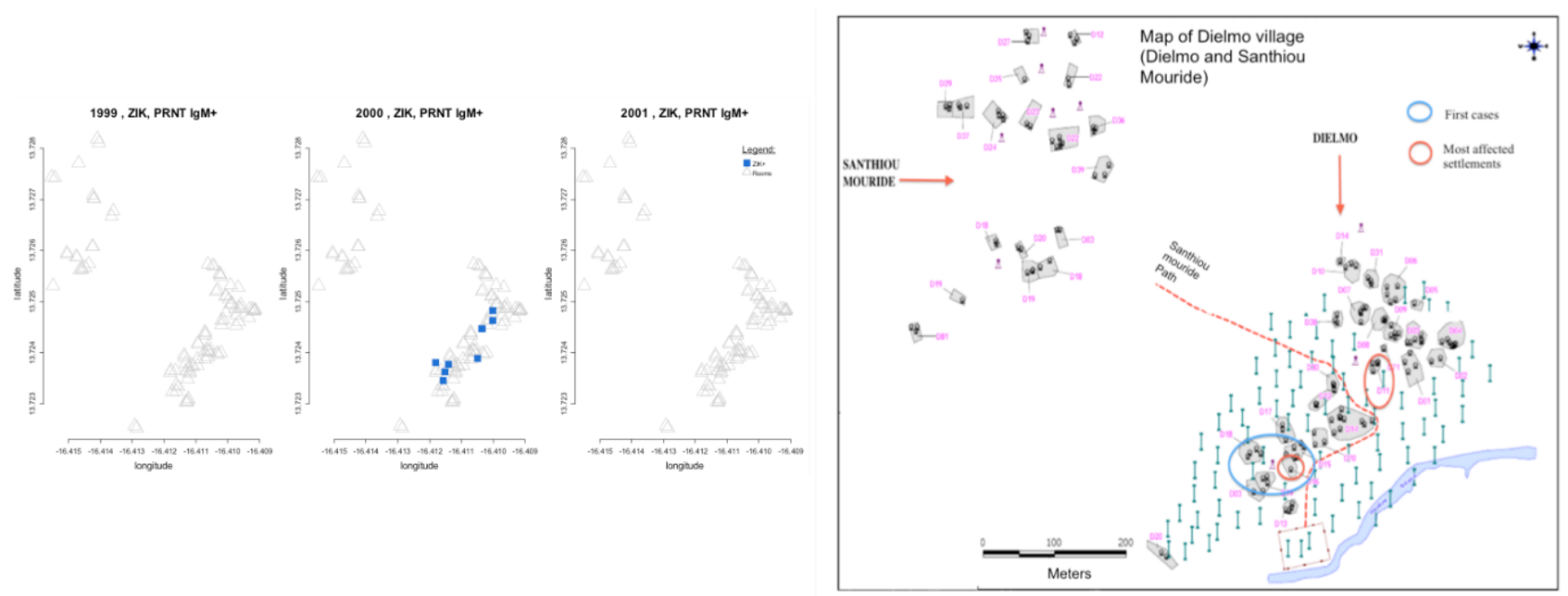

Figure 4. Zika IgM and IgG confirmed cases locations in 1999, 2000 and 2001. 

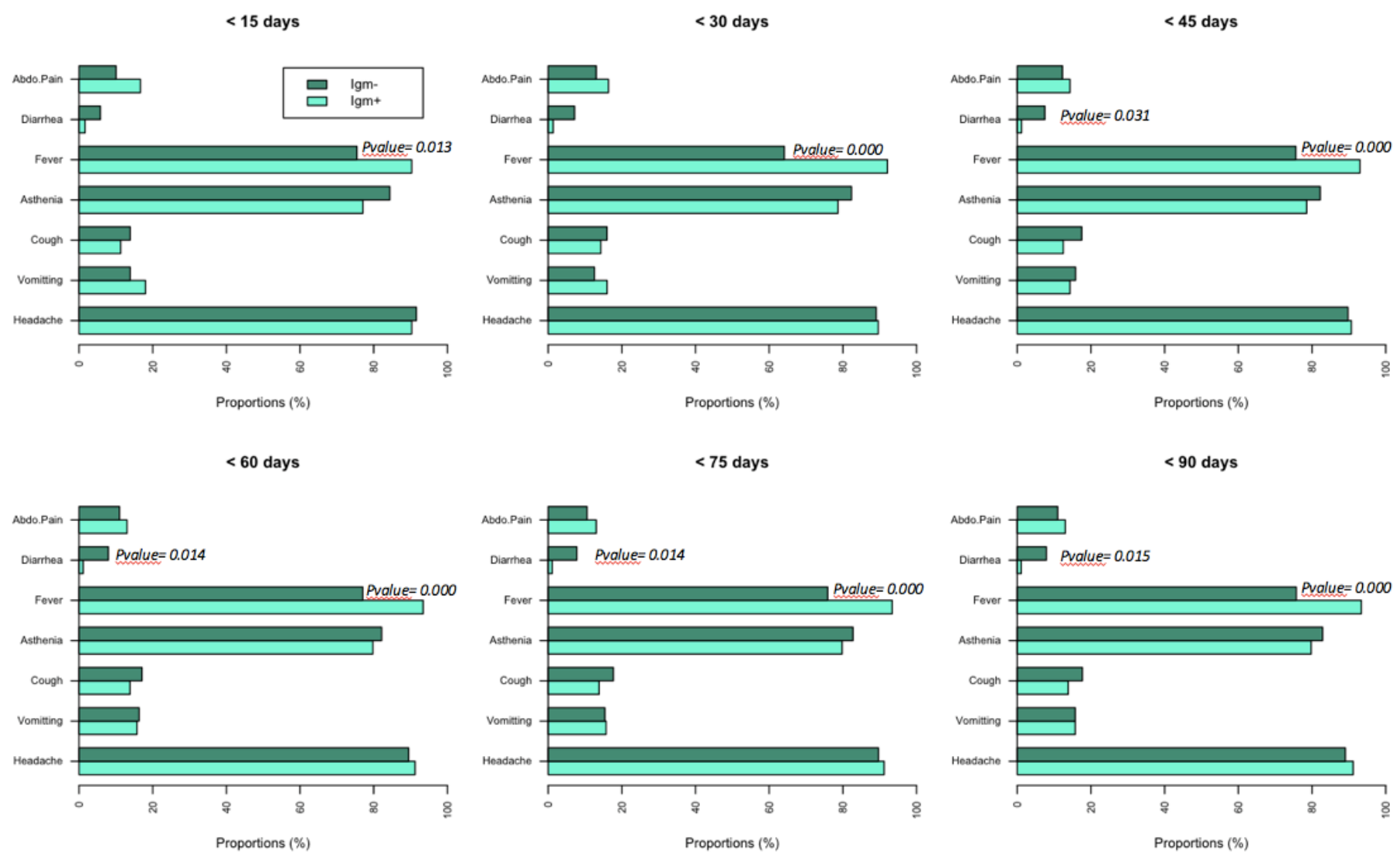

Figure 5. Proportion of clinical symptoms in J-days before the sampling time.

Table 4. Association between number of non-malaria febrile episodes and the Zika outbreak

\begin{tabular}{|l|c|c|c|c|c|c|c|c|}
\hline & & & \multicolumn{7}{|c|}{$\begin{array}{c}\text { Prevalence of Non-Malaria febrile episodes in J } \\
\text { days before the IgM test }\end{array}$} \\
\hline & & & \multicolumn{5}{|c|}{} \\
\hline & $\begin{array}{l}\# \\
\text { indiv }\end{array}$ & $\begin{array}{c}\text { \# febrile } \\
\text { episode }\end{array}$ & $<\mathbf{1 5} \mathbf{j}$ & $<\mathbf{3 0} \mathbf{j}$ & $<\mathbf{4 5} \mathbf{j}$ & $\begin{array}{c}<\mathbf{6 0} \\
\mathbf{j}\end{array}$ & $<\mathbf{7 5} \mathbf{j}$ & $<\mathbf{9 0} \mathbf{j}$ \\
\hline Group1(IgM+) & 13 & 13 & 0.55 & 0.60 & 0.55 & 0.58 & 0.58 & 0.58 \\
\hline Group2(IgM-) & 182 & 182 & 0.27 & 0.36 & 0.43 & 0.46 & 0.50 & 0.51 \\
\hline & & & $4.4 \mathrm{E}-05$ & $2.1 \mathrm{E}-04$ & 0.05 & 0.03 & 0.14 & 0.20 \\
\hline
\end{tabular}

P-values are for comparison of prevalence between Group1 and Group2

(3-5 days) of the infection then disappear after 3 months, while IgG antibodies can persist for years [1].

Cross-reactions in flaviviruses are frequent in areas where multiple flaviviruses are endemic. The plaque reduction neutralization test is highly specific to confirm the serological ELISA analysis and rule out cross-reactivity. Our results showed that among patients with specific neutralizing IgG antibody against ZIKV, in 2000 and in 2001, few had developed specific antibodies against other tested flaviviruses. In 2001, only three patients had multiple seroprevalence, while five patients developed only specific antibodies against ZIKV and not to other tested flaviviruses. Of these three patients, one was detected with higher titer in YFV antibodies than in ZIKV. This could suggest a previous infection with YFV and when exposed to ZIKV the patient developped high levels of YFV neutralizing antibodies. Also, this patient might have received a Yellow fever vaccination that enhanced YFV antibodies. This highlights the endemicity of such flaviviruses in Dielmo, and also increase the serological evidence of the ZIKV outbreak in 2000 as we found up to three-fold dilution differences in neutralizing antibody titers.
Our data suggested that the active transmission of ZIKV was more important in November, period which corresponds to the end of the rainy season, with high density of Aedes mosquitoes due to the presence of temporary ponds. Entomological and virological studies in Senegal reported an increase in the circulation of Aedes mosquitoes commonly associated to ZIKV due to the presence of its breeding sites like artificial water containers in house and in peridomestic areas [16,20]. Geographical repartition of domestic Ae. aegypti in Senegal showed its large distribution in savannah areas which include Dielmo village [30]. Moreover, studies in the Southeast of the country suggested a probable role of Ae. vittatus in ZIKV transmission to humans [16]. Recently, Ae. vittatus and Ae. luteocephalus showed competence to transmit local Zika strain compared to Ae. aegypti populations who were susceptible but did not transmit the virus [31].

The rise in IgG antibodies in 2001 is correlated to a larger previous recent infection and confirmed an under-estimation of the 2000 outbreak. The undiagnosed cases could be partly due to an early sampling with no detectable IgM antibodies in the serum. Moreover, we did not realize PCR tests to directly detect the presence of ZIKV in samples due to the storage conditions, $-20^{\circ} \mathrm{C}$ for more than 17 years. This 2000 ZIKV outbreak could thus be more important and affect a larger portion of Dielmo population.

Our study revealed that children and young adults were most affected by ZIKV. Due to their vulnerable immune system, young children are more susceptible to virus infections than elders. Young adults are more likely involved in daily agriculture activities and are thus more exposed to diurne mosquitoes bites and viral infections. 
We also found that previous exposition to ZIKV was more prevalent in the adult population of Dielmo. Elsewhere in Africa, similar prevalence of ZIKV antibodies in human populations has been reported [14,32]; in Mozambique, the prevalence was higher in adults compared to children [33].

Spatial analysis showed that ZIKV cases were more reported around the river and has extended throughout the village. The dense vegetation areas around the river being favorable to the development of wild Aedes mosquitoes like Ae. vittatus and Ae. luteocephalus that live in close contact with humans [14-16,31], this suggest that the first cases may appear around this specifically location then spread throughout the village, vectors promoting the rapid transmission of the virus.

The virus is probably endemic in Dielmo and circulates in the area even if no epidemics have been reported. With the two recent Polynesia large epidemics, in which co-infections of Dengue and Zika virus have been described it is probable that some ZIKV infections have been misdiagnosed both in vectors and human populations. In Gabon, retrospective studies revealed a ZIKV epidemic masked by concomitant Chikungunya and Dengue outbreaks in 2007 [18]. It would therefore be very likely that ZIKV has circulated in the Senegalese human population however due to the similar clinical presentation with other flaviviruses, the virus circulation has been unnoticed. This highlights the need to develop rapid and specific diagnostic tools for ZIKV detection.

Malaria is holoendemic in Dielmo, with high transmission during all year. The longitudinal Dielmo study reported many cases of nonmalaria fever since the 1990s. In many African areas where malaria is endemic, the development of effective rapid malaria diagnostic tests highlights the increase number of fever of unknown origin mainly in infants. In literature, more than $50 \%$ of fever in these areas were reported to be non-malaria [21]. Interest in probable viral origin due to ZIKV in non-malaria fever in Dielmo has not been reported. Those fevers may be probably caused by viral infections transmitted by Aedes vectors or other mosquitoe species. Our findings showed however a significant association in Zika infection and non-malaria fever. As clinical characteristics, our results suggest that fever could be a symptom associated with Zika infection which is inconsistent with litterature as approximately $80 \%$ of $\mathrm{Zika}$ infection are reported as not associated with fever. However, absence of clinical presentation by the time of sample collection is definitely a limitation to our analysis.

\section{Conclusions}

According to $\mathrm{WHO}$, seroprevalence studies are urgently needed in the recent context of Zika epidemics to better assess the risk of emergency in countries where the virus is endemic. Few data are available about Zika infection in Africa. Our study revealed unnoticed Zika virus epidemic in febrile population of Senegal in 2000. Since Senegal is playing a central role in the evolution and diffusion of the virus [34] and considering that microcephaly epidemic occurred in Cabo Verde a neighboring country, this put together with our findings highlights the importance of ZIKV surveillance in the area. Further studies on ZIKV prevalence and diagnostic tools development should be of major priority in public health programs.

\section{Key issues}

- Flaviviruses infections such as Zika or dengue fever induce similar clinical symptoms with malaria and remain unnoticed in areas where the disease is endemic.
- Few studies in Africa took interest in the origin of these non-malaria febrile illnesses.

- In Senegal, the Dielmo project, a malaria surveillance program in Dielmo village revealed a large proportion of febrile disease of unknown origin.

- We conducted a retrospective study to determine the seroprevalence of Zika virus (ZIKV) in Dielmo in febrile patients in 1999, 2000 and 2001.

- Our study revealed a serological evidence of ZIKV epidemic in Dielmo in 2000 :

o In $2000,8.4 \%(17 / 203)$ samples were tested positive for ZIKV IgM and 6.9\% (14/203) were confirmed by PRNT. No ZIKV IgM positive were found in 1999 and 2001.

o Children and young adults were more affected. There was no ZIKV IgM positive in patients over 50 years old.

o The high ZIKV IgG seroprevalence in the population study $(85.82 \%)$ reflects a regular circulation of the virus in the area.

o We found a strong association between Zika infections and nonmalaria febrile episodes $(p<0.01)$.

- Based on WHO, seroprevalence studies are needed to better assess the risk of emergency in endemic areas. Our results highlights the importance of ZIKV surveillance in Senegal.

\section{Author contributions}

AAS, OF, A T-B, Ousm F, MD designed the study. FB, CL, OF, AAS, OF, RNPNM and MS analyzed the data and wrote the manuscript. FB performed the experiment.

\section{Acknowledgments}

We acknowledge the members of the Unité d'immunologie de l'Institut Pasteur de Dakar and the Unité d'épidémiologie de l'Institut Pasteur de Dakar, Dakar, Senegal for providing samples. We thank all the scientifical research group of the Pôle de Virologie de l'Institut Pasteur de Dakar, Dakar, Senegal, particulary Moussa Dia, Oumar Ndiaye and Rouguietou Sylla.

\section{References}

1. Lanciotti RS, Kosoy OL, Laven JJ, Velez JO, Lambert AJ, et al. (2008) Genetic and serologic properties of Zika virus associated with an epidemic, Yap State, Micronesia, 2007. Emerg Infect Dis 14: 1232-1239. [Crossref]

2. Hayes EB (2009) Zika virus outside Africa. Emerg Infect Dis 15: 1347-1350. [Crossref]

3. Duffy MR, Chen TH, Hancock WT, Powers AM, Kool JL, et al. (2009) Zika virus outbreak on Yap Island, Federated States of Micronesia. N Engl J Med 360: 2536-2543. [Crossref]

4. Oehler E, Watrin L, Larre P, Leparc-Goffart I, Lastère S, et al. (2014) Zika virus infection complicated by Guillain-Barré syndrome - case report, French Polynesia, December 2013. Euro Surveill 19: pii=20720. [Crossref]

5. Kwong JC, Druce JD, Leder K (2013) Zika virus infection acquired during brief travel to Indonesia. Am J Trop Med Hyg 89: 516-517. [Crossref]

6. Zanluca C, Melo VC, Mosimann AL, Santos GI, Santos CN, et al. (2015) First report of autochthonous transmission of Zika virus in Brazil. Mem Inst Oswaldo Cruz 110: 569-572. [Crossref]

7. Sarno M, Sacramento GA, Khouri R, do Rosário MS, et al. (2016) Zika Virus Infection and Stillbirths: A Case of Hydrops Fetalis, Hydranencephaly and Fetal Demise. PLoS Negl Trop Dis 10: e0004517. [Crossref]

8. Fauci AS, Morens DM (2016) Zika Virus in the Americas--Yet Another Arbovirus Threat. N Engl J Med 374: 601-604. [Crossref] 
9. Oliveira Melo AS, Malinger G, Ximenes R, Szejnfeld PO, Alves Sampaio S, et al. (2016) Zika virus intrauterine infection causes fetal brain abnormality and microcephaly: tip of the iceberg? Ultrasound Obstet Gynecol 47: 6-7. [Crossref]

10. Foy BD, Kobylinski KC, Foy JLC, Blitvich BJ, da Rosa AT, et al. (2011) Probable nonvector- borne transmission of Zika virus, Colorado, USA. Emerg Infect Dis 14:880882. [Crossref]

11. Musso D, Roche C, Robin E, Nhan T, Teissier A, et al. (2015) Potential sexual transmission of Zika virus. Emerg Infect Dis 21: 359-361. [Crossref]

12. (2016) World Health Organization. Situation report: Zika virus microcephaly GuillainBarre syndrome. 6 October. WHO.

13. (2016) Pan American Health Organization / World Health Organization. Epidemiological Update: Neurological syndrome, congenital anomalies and Zika virus infection. 17 January, Washington, D.C.: PAHO/WHO.

14. Fagbami $\mathrm{AH}$ (1979) Zika virus infections in Nigeria: virological and seroepidemiological investigations in Oyo State. J Hyg (Lond) 83: 213-219. [Crossref]

15. HADDOW AJ, WILLIAMS MC, WOODALL JP, SIMPSON DI, GOMA LK (1964) TWELVE ISOLATIONS OF ZIKA VIRUS FROM AEDES (STEGOMYIA) AFRICANUS (THEOBALD) TAKEN IN AND ABOVE A UGANDA FOREST. Bull World Health Organ 31: 57-69. [Crossref]

16. Diallo D, Sall AA, Diagne CT, Faye O, Faye O, et al. (2014) Zika virus emergence in mosquitoes in southeastern Senegal, 2011. PLoS One 9: e109442. [Crossref]

17. Wong PS, Li MZ, Chong CS, Ng LC, Tan CH (2013) Aedes (Stegomyia) albopictus (Skuse): a potential vector of Zika virus in Singapore. PLoS Negl Trop Dis 7: e2348. [Crossref]

18. Grard G, Caron M, Mombo IM, Nkoghe D, Mboui Ondo S, et al. (2014) Zika virus in Gabon (Central Africa)--2007: a new threat from Aedes albopictus? PLoS Negl Trop Dis 8: e2681. [Crossref]

19. MACNAMARA FN (1954) Zika virus: a report on three cases of human infection during an epidemic of jaundice in Nigeria. Trans R Soc Trop Med Hyg 48: 139-145. [Crossref]

20. Monlun E, Zeller H, Le Guenno B, Traore-Lamizana M, Hervy JP, et al. (1993) Surveillance of the circulation of arbovirus of medicalinterest in the region of eastern Senegal. Bull Soc PatholExot 86: 21-28.

21. Trape JF, Rogier C, Konate L, Diagne N, Bouganali H, et al. (1994) The Dielmo project: a longitudinal study of natural malaria infection and the mechanisms of protective immunity in a community living in a holoendemic area of Senegal. Am J Trop Med Hyg 51: 123-137.
22. Trape JF, Tall A, Sokhna C, Ly AB, Diagne N, et al. (2014) The rise and fall of malaria in a West African rural community, Dielmo, Senegal, from 1990 to 2012: a 22 year longitudinal study. Lancet Infect Dis 14: 476-488. [Crossref]

23. Socolovschi, C, Mediannikov O, Sokhna C, Tall A, Diatta G, et al. ( 2010) Rickettsia felis-associated uneruptive fever, Senegal. Emerg Infect Dis Jul 16: 1140-1142. [Crossref]

24. Sokhna C, Mediannikov O, Fenollar F, Bassene H, Diatta G, et al. (2013) Point-of-Care Laboratory of Pathogen Diagnosis in Rural Senegal. PLoS Negl Trop Dis 7: e1999. [Crossref]

25. Guzman MG, Kouri G (1996) Advances in dengue diagnosis. Clin Diagn Lab Immunol 3: 621-627.

26. (2004) Manual for the Monitoring of Yellow Fever Virus Infection, WHO, Departmen of Immunization, Vaccines and Biological, Geneva, World Health -Organization, WHO/IVB/04.08)

27. Faye O, Diallo M, Dia I, Ba Y, Mondo M, et al. (2007) [Integrated approach to yellow fever surveillance: pilot study in Senegal in 2003-2004]. Bull. Soc Pathol Exot 100 187-192. [Crossref]

28. De Madrid AT, Porterfield JS (1969) A simple micro-culture method for the study of group B arboviruses. Bull World Health Organ 40: 113-121. [Crossref]

29. Beaty BJ, Calisher CH, Shope RS (1989) Arboviruses. Schmidt NJ, Emmons RW (Edtr). Diagnostic Procedures for Viral, Rick- ettsial and Chlamydial Infections. (6 $6^{\text {th }}$ Edn), Washington, DC: American Public Health Association: 797-856.

30. Huber K, Ba Y, Dia I, Mathiot C, Sall A A, et al. (2008) Aedes aegypti in Senegal genetic diversity and genetic structure of domestic and sylvatic populations. Am J Trop Med Hyg 79: 218-229. [Crossref]

31. Diagne CT, Diallo D, Faye O, Ba Y, Faye O, et al. (2015) Potential of selected Senegalese Aedes spp. mosquitoes (Diptera: Culicidae) to transmit Zika virus. BMC Infectious Diseases 15: 492. [Crossref]

32. Fokam EB, Levai LD, Guzman H, Amelia PA, Titanji VP, et al. (2010) Silent circulation of arboviruses in Cameroon. East Afr Med J 87: 262-268. [Crossref]

33. Gudo ES, Falk KI, Ali S, Muianga AF, Monteiro V, et al. (2016) A Historic Report of Zika in Mozambique: Implications for Assessing Current Risk. PLoS Negl Trop Dis 10: e0005052. [Crossref]

34. Shen S, Shi J, Wang J, Tang S, Wang H, et al. (2016) Phylogenetic analysis revealed the central roles of two African countries in the evolution and worldwide spread of Zika virus. Virol Sin 31: 118-130. [Crossref]

Copyright: (C2018 Fatim Ba. This is an open-access article distributed under the terms of the Creative Commons Attribution License, which permits unrestricted use, distribution, and reproduction in any medium, provided the original author and source are credited. 